\title{
Transcriptional activation of endoplasmic reticulum chaperone GRP78 by HCMV IE1-72 protein
}

Derick Shi-Chen $\mathrm{Ou}^{1,2}$, Sung-Bau Lee ${ }^{2, *}$, Chi-Shuen $\mathrm{Chu}^{2,{ }^{*}}$, Liang-Hao Chang ${ }^{2}$, Bon-chu Chung ${ }^{1,4}$, Li-Jung Juan ${ }^{2,3}$

${ }^{1}$ Institute of Molecular and Cellular Biology, National Tsing Hua University, 101, Section 2, Kuang-Fu Road, Hsinchu 300; ${ }^{2}$ Genomics Research Center, Academia Sinica, 128 Academia Road, Section 2, Nankang, Taipei 115; ${ }^{3}$ Institute of Molecular Medicine, National Taiwan University, No.7, Chung San South Road, Taipei 100; ${ }^{4}$ Institute of Molecular Biology, Academia Sinica, 128 Academia Road, Section 2, Nankang, Taipei 115

Glucose-regulated protein 78 (GRP78), a key regulator of endoplasmic reticulum (ER) stress, facilitates cancer cell growth and viral replication. The mechanism leading to $\operatorname{grp} 78$ gene activation during viral infection is largely unknown. In this study, we show that the immediate-early 1 (IE1-72) protein of the human cytomegalovirus (HCMV) is essential for HCMV-mediated GRP78 activation. IE1-72 upregulated grp78 gene expression depending on the ATPbinding site, the zinc-finger domain and the putative leucine-zipper motif of IE1-72, as well as the ER stress response elements (ERSEs) on the grp 78 promoter. The purified IE1-72 protein bound to the CCAAT box within ERSE in vitro, whereas deletion mutants of IE1-72 deficient in grp78 promoter stimulation failed to do so. Moreover, IE1-72 binding to the $\operatorname{grp} 78$ promoter in infected cells accompanied the recruitment of TATA box-binding protein-associated factor 1 (TAF1), a histone acetyltransferase, and the increased level of acetylated histone $\mathrm{H4}$, an indicator of activestate chromatin. These results provide evidence that HCMV IE1-72 activates grp 78 gene expression through direct promoter binding and modulation of the local chromatin structure, indicating an active viral mechanism of cellular chaperone induction for viral growth.

Keywords: GRP78; ER stress elements; HCMV; IE1-72; TAF1

Cell Research (2011) 21:642-653. doi:10.1038/cr.2011.10; published online 11 January 2011

\section{Introduction}

Endoplasmic reticulum (ER) stresses, such as accumulation of misfolded proteins, calcium depletion and

\footnotetext{
*These two authors contributed equally to this work. Correspondence: Bon-chu Chung ${ }^{\mathrm{a}}$, Li-Jung Juan ${ }^{\mathrm{b}}$ ${ }^{\mathrm{a}}$ Tel: +886-2-2789-9215; Fax: +886-2-27826085

E-mail: mbchung@sinica.edu.tw

${ }^{\text {b}}$ Tel: +886-2-27871234; Fax: +886-2-27898811

E-mail: 1jjuan@gate.sinica.edu.tw

Abbreviations:GRP78 (glucose-regulated protein 78); HCMV (human cytomegalovirus); IE1-72 (immediate-early protein 1-72); ER (endoplasmic reticulum); UPR (unfolded protein response); TAF1 (TAF1 RNA polymerase II, TATA box-binding protein-associated factor, $250 \mathrm{kDa}$ ); $\mathrm{Ab}$ (antibody); MOI (multiplicity of infection); d.p.i. (days post infection); h.p.i. (hours post infection); EMSA (electrophoretic mobility shift assay); ChIP (chromatin immunoprecipitation); siRNA (small interfering RNA)

Received 9 June 2010; revised 6 September 2010; accepted 21 September 2010; published online 11 January 2011
}

glycosylation block [1], trigger the unfolded protein response (UPR) through multiple signaling pathways $[1,2]$. UPR includes the upregulation of a family of chaperones called glucose-regulated proteins (GRPs). Among them, glucose-regulated protein 78 (GRP78), which resides in the $\mathrm{ER}$, is a $78 \mathrm{kDa}$ protein that aids protein folding in the ER [2]. Under normal condition, GRP78 binds to three UPR sensors, ER to nucleus signaling 1 (IRE1) [3], activating transcription factor 6 (ATF6) [4] and PKR-like ER kinase (PERK) [3], and prevents their activation and transmission of UPR signaling into the nucleus. Upon UPR, GRP78 changes its binding preference toward unfolded proteins, releasing the three sensors to initiate the UPR signaling. IRE1 and ATF6 $[4,5]$ have been demonstrated to induce most sets of genes in the UPR, whereas PERK phosphorylates the eukaryotic translation initiation factor $2 \alpha$ subunit (eIF $2 \alpha$ ), resulting in a translational block [6]. The activated IRE1 undergoes dimerization, and processes the mRNA of the X-box-binding protein 
1 (XBP-1) to a mature form that encodes a functional transcription factor to induce the expression of proteins involved in ER-associated degradation proteins [3, 7]. After being released from GRP78, ATF6, an ER transmembrane protein, is transported to the Golgi and proteolytically cleaved. The cleavage releases the cytosolic $\mathrm{N}$-terminus of ATF6 (N-ATF6), which then is transported into the nucleus, and serves as a transcriptional factor to induce chaperone genes including GRP78 [8]. N-ATF6 binds to the ER stress response elements (ERSEs) of the grp 78 promoter $[9,10]$; ERSE is a tripartite structure with nine GC-rich nucleotides flanked by two CCAAT boxes [10]. N-ATF6 binds to one CCAAT box, and YY1 binds to the other [9]. Many other factors bind to ERSEs as well. For example, nuclear transcription factor $\mathrm{Y}$ prefers the CCAAT box of ERSE [11]. In contrast, SP1 and general transcription factor II-I $\left(\mathrm{TF}_{\mathrm{III}}\right)$ recognize the middle region of the ERSE $[12,13]$.

Viral infection has been shown to elicit the UPR as well $[14,15]$, and many viruses modulate the UPR signaling pathways to facilitate their growth [14]. Buchkovich et al [16] showed that depletion of GRP78 impairs the assembly of human cytomegalovirus (HCMV). The same laboratory previously demonstrated that HCMV infection blocks ATF6 cleavage and eIF2 phosphorylation [17]. Although the mature form of $x b p-1$ is observed in HCMV-infected cells, its downstream genes, such as the ER degradation-enhancing $\alpha$-mannosidase-like protein, can not be activated [17]. Interestingly, in the absence of the active form of ATF6, HCMV infection still induces GRP78 synthesis [17]. How HCMV upregulates GRP78 expression without activating ATF6 remains unclear.

HCMV is a widely spread pathogen carried by $70 \%$ $90 \%$ of the world's population. HCMV-mediated diseases occur mostly in immunosuppressed patients, such as those with acquired immune deficiency syndrome or organ transplants [18]. HCMV infection accompanies the expression of a variety of viral and cellular genes under the control of the viral immediate-early (IE) proteins IE1-72 and IE2-86. IE1-72 and IE2-86 are alternatively spliced transcripts driven by the major IE promoter (MIEP) [19]. They associate with diverse cellular proteins. For example, IE1-72 complexes with nuclear factor I/C (NF1/CTF1) and transactivates the TATA-less promoter of DNA polymerase $\alpha$ [20]. When interacting with p107, IE1-72 relieves the inhibition of cyclin E/ cdk2 kinase as well as PML-mediated repression [21, 22]. IE1-72, as a kinase, transactivates E2F-dependent promoters by phosphorylating both $\mathrm{pRb}$ and E2F members [23]. Moreover, IE1-72 may also induce the AP1dependent gene expression via the mitogen activated protein kinase (MAPK)/extracellular signal regulated kinase
(ERK) pathway [24]. Control of these cell cycle-related genes indicates that IE1-72 has the potential to cause cell transformation, as reported by Cobbs et al. [25-27], who showed that glioma cells are transformed by ectopic expression of IE1-72.

In this study, we investigated the regulation of $\operatorname{grp} 78$ expression during HCMV infection. We confirmed that HCMV infection induces GRP78 via IE1-72-mediated transcriptional control, and that IE1-72 binds to the grp78 promoter in vitro and in infected cells. Important domains within IE1-72 and the essential region in the grp 78 promoter responsible for the activation were also identified. Furthermore, we found that IE1-72 binding accompanies the recruitment of a transcriptional co-regulator with intrinsic histone acetyltransferase activity and the increase of local histone acetylation. These results suggest that IE1-72 regulates grp 78 expression by modulating the chromatin structure.

\section{RESULTS}

\section{HCMV induces grp78 expression through promoter regu- lation}

To confirm the induction of GRP78 during HCMV infection, mock- or HCMV-infected fibroblasts were harvested, followed by western blot analysis using antibody (Ab) against GRP78. As shown in Figure 1A, GRP78 was induced 1 day post infection (d.p.i.; compare lane 4 with lane 3 ) and the level was gradually increased up to 3 d.p.i. (lanes 6 and 8). As controls, the two IE proteins of HCMV, IE1-72 and IE2-86, were expressed by 1 d.p.i. These results confirm previous findings that GRP78 is induced in HCMV-infected cells [17].

Whether GRP78 induction by HCMV is determined at the mRNA level was analyzed. Consistently, the mRNA level of $\operatorname{grp} 78$ was increased in cells 1 d.p.i., and accumulated through 3 d.p.i. (Figure 1B). The increase of grp 78 mRNA in lane 5 in the absence of HCMV indicates that $\operatorname{grp} 78$ expression is altered by cellular factors during cell culture, and this is independent of HCMV infection. The grp 78 mRNA induction also occurred in a viral dose-dependent manner (Figure 1C). In contrast, the mRNA level of the protein disulfide isomerase (PDI), another ER-resident protein, was not altered upon HCMV infection (Figure 1B and 1C). These data demonstrate the specificity of HCMV-induced grp 78 expression. We further analyzed whether the induction of $g r p 78$ by HCMV was achieved through promoter regulation. Fibroblasts were transfected with the luciferase reporter construct driven by the human grp 78 promoter, followed by HCMV infection. The luciferase activity from cells 1 d.p.i. was enhanced proportionally to the viral titer, 
as compared with that from mock-infected cells (Figure 1D). Together, these results suggest that HCMV infection stimulates grp 78 expression through promoter regulation.

\section{grp 78 induction by HCMV IE 1-72}

Previous studies have suggested that HCMV-mediated GRP78 induction is independent of the UPR pathway [17]. We suspected that viral proteins might directly regulate grp 78 expression. Given that HCMV IE proteins are important transcriptional regulators, we sought to determine whether IE proteins are involved in GRP78 regulation. First, U2OS cells were transiently transfected with the HCMV IE1-72 or IE2-86 plasmid. At $48 \mathrm{~h}$ post transfection, the level of endogenous grp 78 mRNA was measured using quantitative reverse transcription poly-

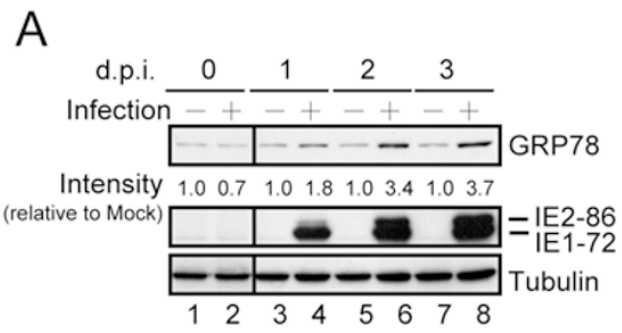

B

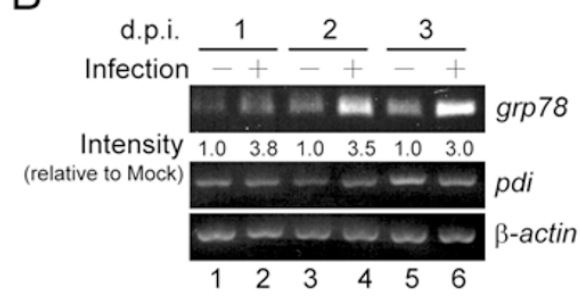

C
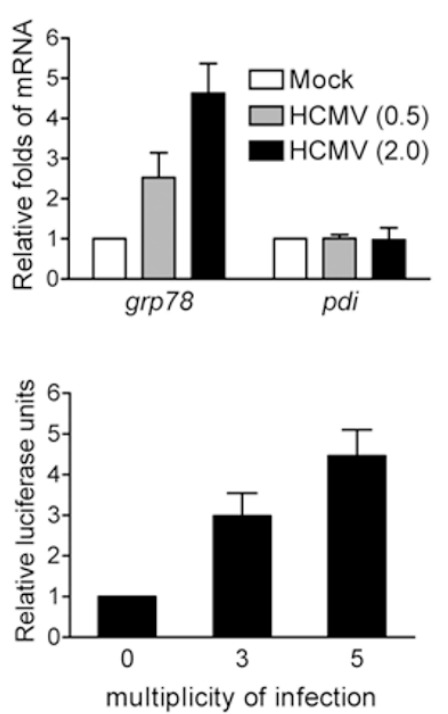

merase chain reaction (qRT-PCR). As shown in Figure 2A, IE1-72 upregulated the grp78 mRNA level by approximately 15-fold, whereas IE2-86 had little effect. Consistently, the protein level of GRP78 was increased only in the presence of IE1-72 (Figure 2B). IE2-86, even at a higher protein level, caused only limited induction of GRP78 (Figure 2B). To address the specificity of IE172 on GRP78 induction, the levels of GRP78, GRP94 and PDI were monitored in the presence of increasing amounts of ectopically expressed IE1-72. As shown in Figure 2C, the level of GRP78, but not GRP94 or PDI, was increased in an IE1-72 dose-dependent manner. This finding indicates that IE1-72 specifically activated GRP78, and the activation was unlikely through ER stress signaling, because two other ER stress signaling indicators, GRP94 and PDI, were not induced at the same time as GRP78. Next, to ascertain whether IE1-72 is essential for GRP78 induction during infection, the GRP78 protein level was analyzed in HCMV-infected HEL299 cells with scrambled siRNA or siRNA targeting IE1-72 (si-IE1-72). As shown in Figure 2D, 90\% of IE1-72 was successfully knocked down in HCMV-infected cells containing si-IE1-72 (compare lane 4 with lane 2). Depletion of IE1-72 by siRNA did not affect the expression of IE286 , an alternative splice product from the same mRNA. Importantly, the 7.8-fold induction of GRP78 by HCMV

Figure $1 \mathrm{HCMV}$ infection induces grp78 expression at the transcriptional level. (A) HCMV infection increases the GRP78 protein level. Human fibroblasts were mock infected $(-)$ or infected with $\mathrm{HCMV}(+)$ at a multiplicity of infection (MOI) of 1 . At the indicated day post infection (d.p.i.), cells were harvested and subjected to western blot analysis using Abs against the indicated proteins. Mab810 was used to detect both IE1-72 and IE2-86 because it recognizes an epitope shared by both proteins. (B) HCMV upregulates grp78 mRNA level. Total RNA from mockinfected (-) or HCMV-infected (+) MRC-5 cells at the indicated day post infection (dpi) were analyzed by reverse-transcription $\mathrm{PCR}$ with specific primers amplifying grp78, pdi, or $\beta$-actin. $\beta$-actin was used as a loading control. The relative protein and mRNA levels of GRP78 in HCMV-infected cells to those in mock-infected cells were quantified by Quantity One software (BioRad Laboratories) and shown in A and B, respectively. (C) The HCMV dose-dependent accumulation of grp78 mRNA in infected cells. MRC-5 cells were mock infected (white bar) or infected with $\mathrm{HCMV}$ at an MOI of 0.5 (gray bar) or 2.0 (black bar) and harvested at 1 d.p.i. The mRNAs were analyzed by quantitative RT-PCR with primers against grp78 or pdi. (D) HCMV activates the grp78 promoter. MRC-5 cells were transfected with plasmid encoding the firefly luciferase reporter gene driven by the human grp78 promoter. After $24 \mathrm{~h}$, cells were infected with $\mathrm{HCMV}$ at the indicated $\mathrm{MOI}$. Cell lysates were collected at 1 d.p.i. and measured for luciferase activity. Data in $\mathbf{C}$ and $\mathbf{D}$ are presented as the mean \pm SD from three independent assays. 

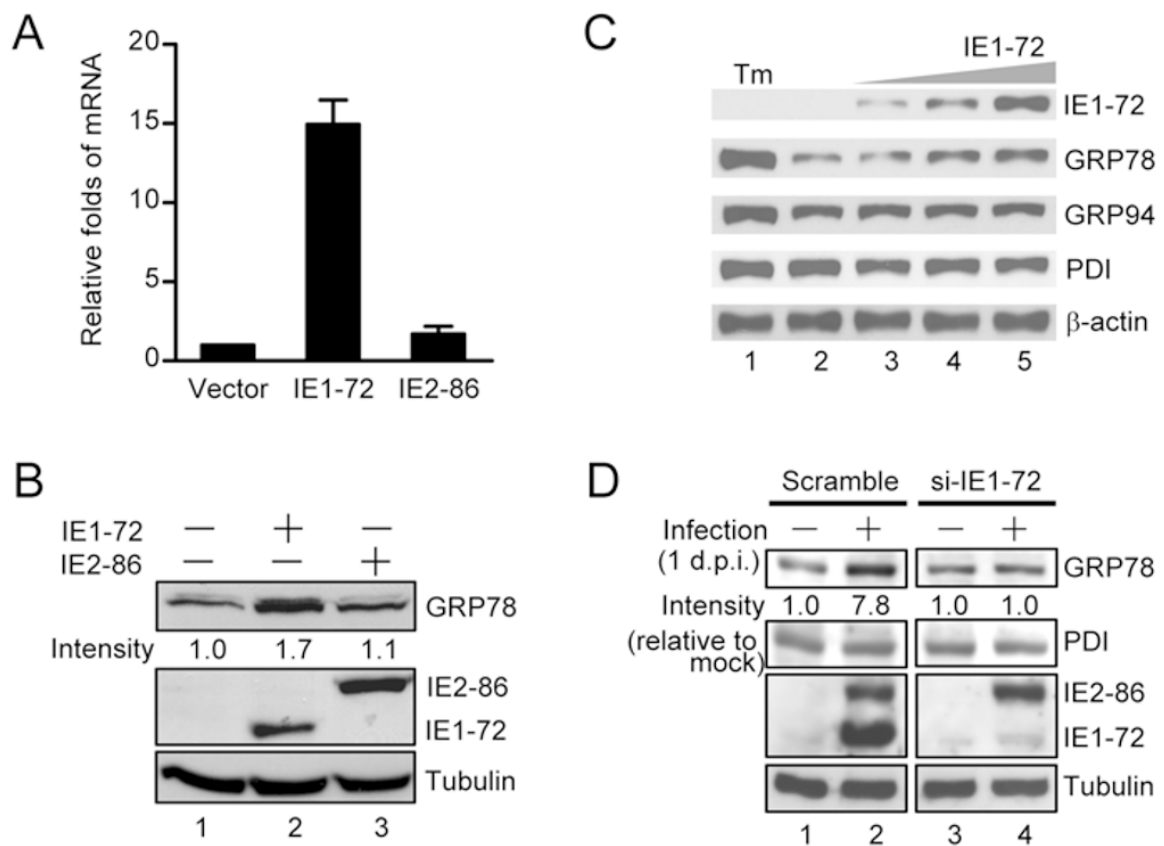

Figure 2 IE1-72, but not IE2-86, upregulates grp78 expression. Ectopic expression of IE1-72 upregulates grp78 mRNA (A) and protein (B) levels. U2OS cells were transiently transfected with vector alone or plasmid encoding IE1-72 or IE2-86, followed by mRNA analysis and western blot at $48 \mathrm{~h}$ post transfection. Tubulin was used as a loading control. (C) IE1-72 specifically increases the protein level of GRP78, but not GRP94 or PDI. U2OS cells were transiently transfected with plasmid encoding IE1-72 in different dosages. After $24 \mathrm{~h}$, cells were harvested and subjected to western blot analysis with Ab against the indicated protein. Tunicamycin $(\mathrm{Tm}, 5 \mu \mathrm{g} / \mathrm{ml})$ was added $16 \mathrm{~h}$ before the cells were harvested. $\beta$-actin was used as a loading control. (D) Knockdown of IE1-72 abolishes HCMV-mediated GRP78 induction. HEL299 cells transiently transfected with scrambled siRNA or si-IE1-72 were mock infected $(-)$ or infected with HCMV $(+)$ at an MOI of 2. Cells were harvested at 1 d.p.i. and subjected to western blot analysis. Tubulin was used as a loading control. Data in $\mathbf{A}$ are presented as the mean \pm SD from three independent assays. The intensity of the bands in B and D was quantified by Quantity One software (BioRad Laboratories).

(compare lane 2 with lane 1) was abolished in the IE172-knocked down cells (compare lane 4 with lane 3). These results suggest that IE1-72, but not IE2-86, is essential for maintaining and regulating GRP78 induction during HCMV infection.

The ATP-binding site, putative zinc-finger and leucinezipper domain of IE1-72 in grp78 promoter transactivation

We continued to investigate whether IE1-72 stimulated grp 78 promoter activity and, if so, which region of IE1-72 was responsible for the transactivation. Figure 3A shows the schematic domain structure of IE1-72. U2OS cells were transfected with the grp 78 promoterdriven luciferase reporter plasmid, together with a plasmid encoding full-length IE1-72 or its internal deletion mutant, and the luciferase activities were measured $24 \mathrm{~h}$ post transfection. As shown in Figure 3B, the full-length IE1-72 activated the grp 78 promoter by approximately 5-fold, whereas its mutant lacking amino acids (aa) 132274 or 290-320 (putative leucine-zipper motif) lost the ability of promoter activation. This observation is consistent with previous reports that these two IE1-72 mutants no longer transactivate the DNA polymerase $\alpha$ promoter [20]. Deletion of the ATP-binding site (aa 173-194) or aa 267-286 (putative zinc-finger motif) also abolished the transactivation ability of IE1-72 on the grp 78 promoter (Figure 3B). In contrast, the mutant lacking aa 421-491 retained the transactivation ability (Figure $3 \mathrm{~B}$ ). These results suggest that the middle part of exon 4 of IE1-72 is important for IE1-72 transactivation function. We also found that IE1-72 mutants with amino acid substitutions within exon 2 or 3 still retained the transactivation potential, although to a lesser extent (Figure 3C, M12, M131 and M13-2). Figure 3D shows the protein levels of the transiently expressed wild-type (WT) and mutant IE172 used above, indicating that the failure of certain mutants to activate the grp 78 promoter was not due to low 
protein expression in cells. These results suggest that the ATP-binding motif (aa 173-194), the putative zinc-finger (aa 267-286) and the leucine-zipper domain (aa 290-320) of IE1-72 are required to upregulate the $\operatorname{grp} 78$ promoter activity.

\section{IE1-72 activates the grp78 promoter through ERSE}

The grp 78 promoter contains three ER stress response elements (ERSEs) [10]. ERSE, a tripartite structure containing $5^{\prime}$-CCAAT $\left(\mathrm{N}_{9}\right)$ CCACG-3', is critical in the regulation of grp 78 promoter activity upon UPR [10]. Mutations within the CCAAT or CCACG motif of ERSE totally abolish the promoter responsiveness to UPR [10]. In this study, we tested whether ERSE also plays an important role in IE1-72-mediated grp 78 promoter activity. U2OS cells were transiently transfected with the luciferase reporter plasmid driven by the WT (Figure 4A) or mutant grp 78 promoter with base substitutions within CCAAT and CCACG motifs of ERSEs (Figure 4A, MMM) with or without IE1-72 expression. Interestingly, the promoter responsiveness to IE1-72 was lost when the ERSEs were disrupted (Figure 4A). These results suggest that IE1-72 was able to elicit its effect through these ERSE sites. To further clarify which region of the ERSE was important for responsiveness to IE1-72, we fused the most adjacent ERSE (sequences illustrated in Figure 4B), with or without mutations, to an SV40 early promoter-driven luciferase reporter and monitored the response to IE1-72. In agreement with previous reports [19, 28], IE1-72 slightly activated SV40 promoter with no ERSE (Figure 4C). The activity was enhanced when the SV40 promoter was fused with the WT ERSE (Figure 4C). This implies that the ERSE indeed mediated IE1-72 function. Importantly, the IE1-72-specific effect was abolished when the left CCAAT box (LM and MM), but not the right CCACG box (RM) or the middle sequence (IM), in the ERSE was mutated (Figure 4C). These results suggest that IE172 mediates grp 78 promoter activation through the left CCAAT box in the ERSE.

\section{Binding of IE1-72 to ERSE}

Subsequently, we explored the mechanism by which IE1-72 activated the grp 78 promoter. IE1-72 protein sequence analysis using DP bind, a web server for sequence-based predictions of DNA-binding residues in DNA-binding proteins $[29,30]$, identified at least four
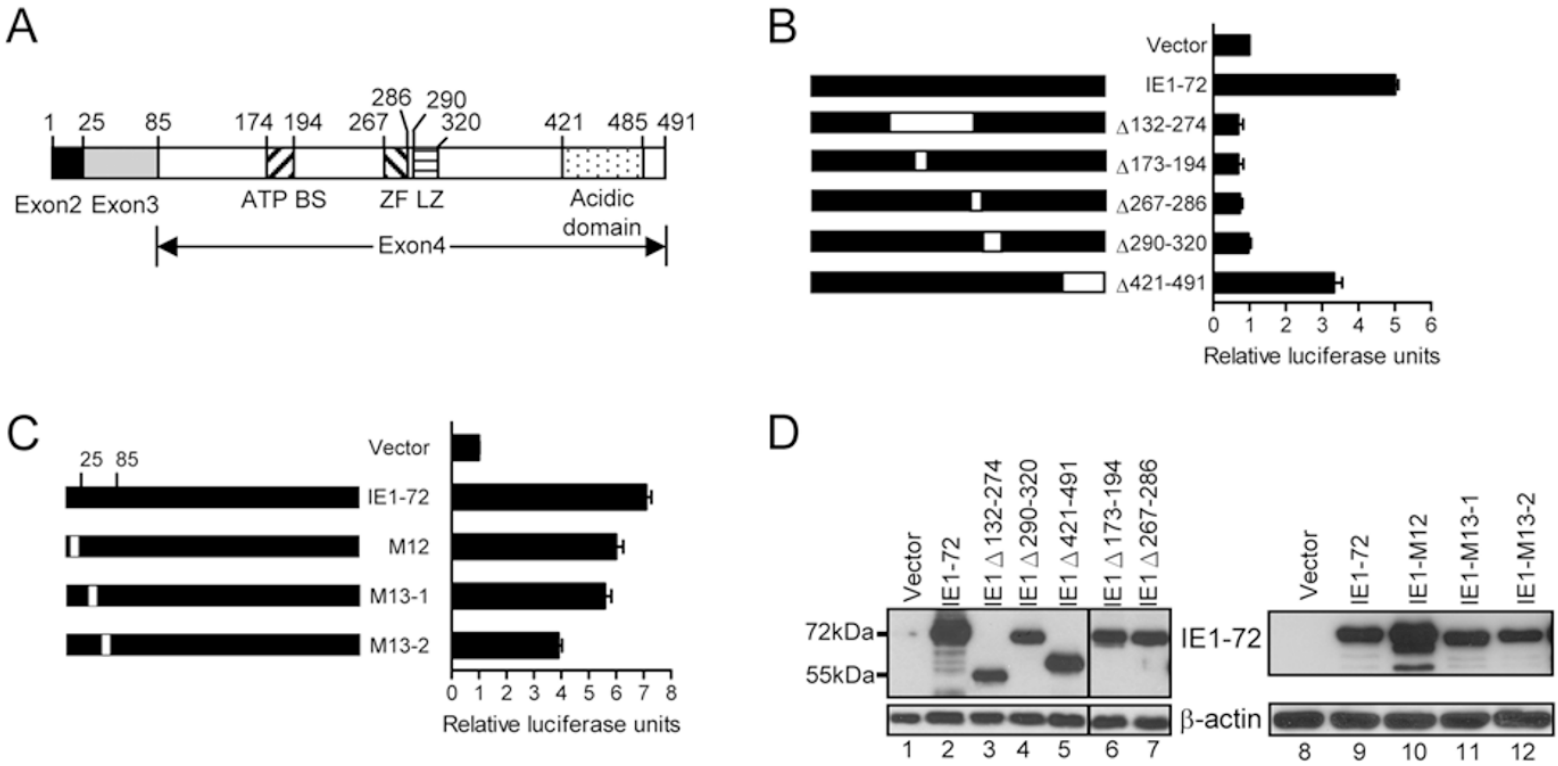

Figure 3 The ATP-binding site, zinc-finger and leucine-zipper motif of IE1-72 are required for grp78 promoter transactivation. (A) A schematic map of full-length IE1-72. ATP BS: ATP-binding site. ZF: zinc-finger motif. LZ: putative leucine-zipper motif. (B) ATP-binding site, zinc finger and leucine-zipper domain are required for IE1-72 to transactivate grp78 promoter. U2OS cells were transfected with plasmid encoding the firefly luciferase gene driven by the human grp78 promoter together with plasmid encoding full-length IE1-72 (1-491) or its deletion mutant lacking indicated amino acids. Cell lysates were harvested and measured for luciferase activity at $48 \mathrm{~h}$ post transfection. (C) Exon 2 and exon 3 are dispensable for IE1-72-mediated grp78 promoter transactivation. Similar experiments as in B were performed, except that cells were transfected with plasmid encoding an IE1-72 mutant with substitutional mutations within exon 2 or exon 3 (M12 on exon 2 or M13-1 and M13-2 on exon 3). Data in $\mathbf{B}$ and $\mathbf{C}$ are presented as the mean \pm SD from three independent assays. (D) Western blots show the protein expression levels of IE1-72 and its mutants used in B and C. $\beta$-actin was used as a loading control. 

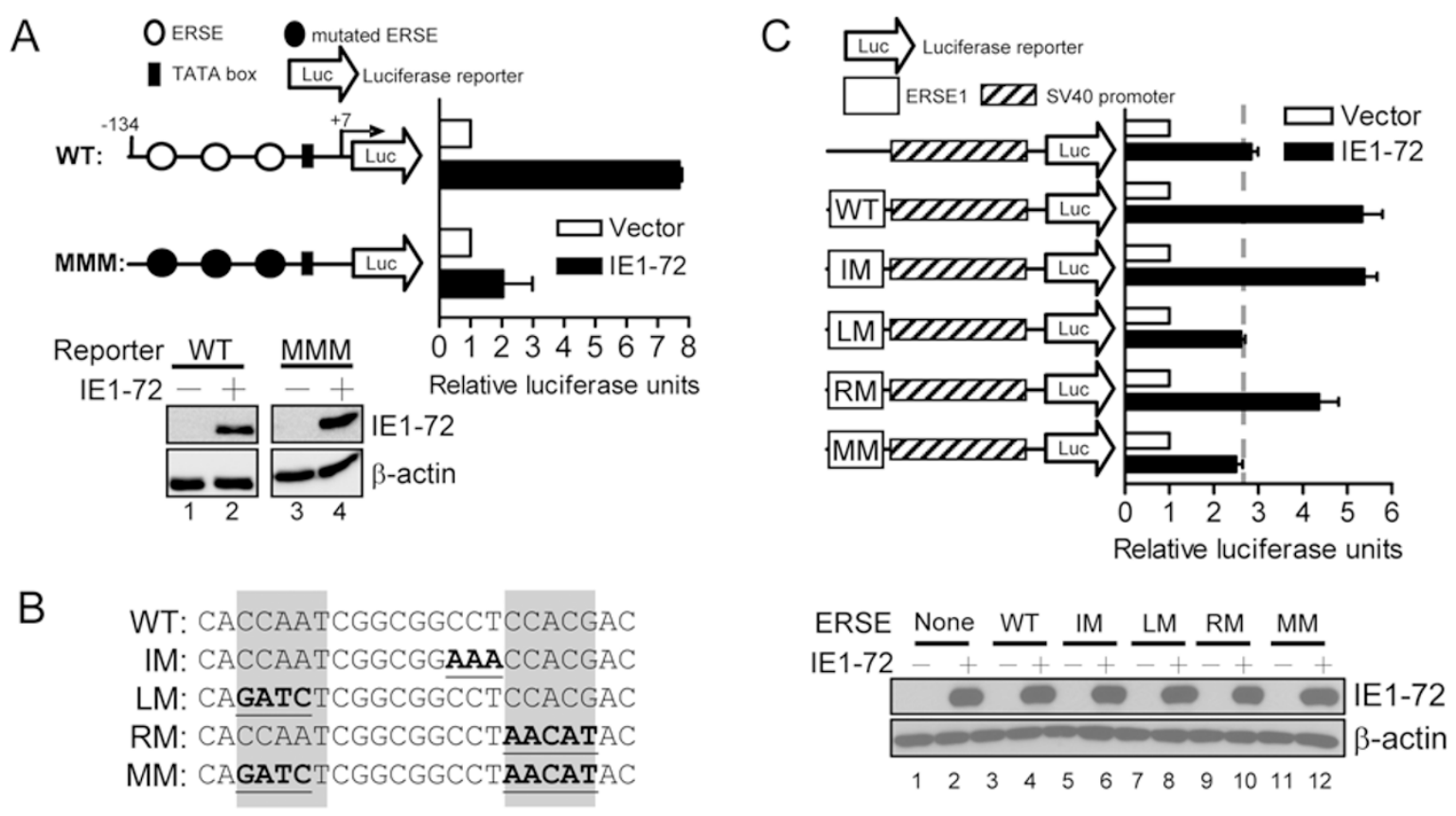

Figure 4 IE1-72 activates the grp78 promoter in an ERSE-dependent manner. (A) ERSE is required for IE1-72 to activate the grp78 promoter. U2OS cells were transfected with plasmid encoding the firefly luciferase gene driven by the wild-type or ERSE-mutated human grp78 promoter together with vector control (white bar) or plasmid encoding IE1-72 (black bar). After $24 \mathrm{~h}$, cells were harvested and measured for luciferase activity. Open oval: ER stress response elements (ERSE). Closed oval: mutated ERSE. Closed square: TATA box. Arrow: the luciferase reporter gene. The western blot below the cartoon of promoters indicates the protein level of IE1-72 in each transfection. $\beta$-actin is used as a loading control. (B) Nucleotide sequences of the wild-type and mutated ERSE1 from -65 to -39 nucleotide of the human grp78 promoter. Mutated nucleotides were bolded and underlined. These ERSEs were fused with the SV40 promoter in the pGL3-luciferase construct as described in Materials and Methods and illustrated in C. (C) IE1-72-mediated grp78 promoter activation requires the left CCAAT box of ERSE. U2OS cells were transfected with the plasmid encoding the firefly luciferase gene driven by the illustrated promoter together with vector control (white bar) or the plasmid encoding IE1-72 (black bar). After $24 \mathrm{~h}$, cells were harvested and measured for luciferase activity. Arrow: the luciferase reporter gene. Open square: ERSE1. Stripped square: SV40 promoter. The western blot below the chart shows the expression of IE1-72 in each transfection. $\beta$-actin was used as a loading control. Data in $\mathbf{A}$ and $\mathbf{C}$ are presented as mean \pm SD from three independent assays.

potential DNA-binding motifs in exon 4 from aa 179 to aa 329. Given that exon 4 of IE1-72 and the ERSE of grp78 promoter were found to be required for IE1-72mediated $\operatorname{grp} 78$ promoter activation (Figures 3 and 4), we examined, using the electrophoretic mobility shift assay (EMSA), whether IE1-72 binds directly to the ERSE. As shown in Figure 5A, the His-tagged IE1-72 (His-IE1-72) expressed in and purified from Escherichia coli binds to the ERSE oligonucleotide, as evidenced by a band shift (compare lane 2 with 1). The intensity of the band was increased depending on the dose of IE172 (lanes 2 to 4), and the band could be super shifted by increasing the amounts of anti-His Ab (lanes 5 to 7). Furthermore, IE1-72 binding to the ERSE was outcompeted by adding 50- or 100 -fold excess cold probe containing the intact ERSE sequence (Figure 5B, compare lanes 5 and 6 with lane 4), but not by probes with base substitu- tions in the left CCAAT box (Figure 5C, compare lanes 4 and 5 with lane 3 ). These results clearly indicate that His-IE1-72 is a DNA-binding protein that specifically recognizes the ERSE. To further examine whether ERSEbinding ability was essential for IE1-72 to activate the grp 78 promoter, we tested whether IE1-72 mutants lacking part of exon 4 essential for $g r p 78$ promoter activation (Figure 3B) were defective in ERSE binding. As shown in Figure 5D, the WT IE1-72, but not mutants lacking part of exon 4, was able to shift the biotinylated probe (compare lanes 5 to 13 with lanes 1 to 4 ). The failure of the IE1-72 mutants to bind the ERSE was not due to a problem in protein expression, as evidenced by the SDSPAGE analysis shown in Figure 5E. These data indicate that the DNA-binding ability is indeed required for IE172 to activate the $\operatorname{grp} 78$ promoter and that the IE1-72 region from aa 132 to aa 286 mediates the DNA binding. 
IE1-72 binding to grp78 promoter accompanies TATA box-binding protein-associated factor 1 (TAF1) recruitment and local histone acetylation

The DNA-binding ability of IE1-72 was further analyzed in vivo. Cells were transfected with a WT or ERSE-substituted reporter together with an empty vector or plasmid encoding IE1-72, followed by chromatin immunoprecipitation (ChIP) assays using an anti-IE Ab to pull down ectopically expressed IE1-72 and the associated chromatin. The precipitated DNA was analyzed by quantitative PCR, with primers specifically amplifying the exogenously added grp 78 promoters from the reporters. As shown in Figure 6A, IE1-72 associated with the WT grp 78 promoter (WT) but not the ERSE-substituted promoter (MMM). This result supports our EMSA data, which show that IE1-72 binds to the ERSE (Figure 5). The binding of IE1-72 to the endogenous grp 78 promoter, but not the $p d i$ promoter, was also confirmed (Figure
$6 \mathrm{~B}$, gray bars). Moreover, the binding was lost when the IE1-72 mutant lacking the ERSE-binding domain (aa 132 to aa 274) was expressed in cells (Figure 6B, black bars). These ChIP results indicate that IE1-72 indeed binds to the $\operatorname{grp} 78$ promoter through the ERSE in cells.

The mechanism by which IE1-72 binding to the ERSE activates the grp 78 promoter was further explored. Because IE1-72 has been reported to bridge the $\mathrm{TF}_{\mathrm{II}} \mathrm{D}$ complex required for transcriptional activation and to rescue the transcriptional defect caused by a mutant TAF1 [31], a component of the $\mathrm{TF}_{\mathrm{II}} \mathrm{D}$ complex and a histone acetyltransferase [32], we monitored if TAF1 associated with the grp 78 promoter concomitantly with IE1-72 binding. MRC-5 cells were mock infected or infected by HCMV, and cells were collected at $24 \mathrm{~h}$ post infection (h.p.i.), and subjected to ChIP assay using IgG or Ab against HCMV IE proteins or TAF1, followed by qPCR amplification of the ERSE region of the $g r p 78$ promoter. As
A

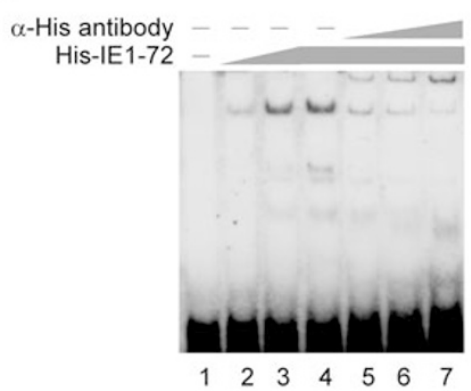

D

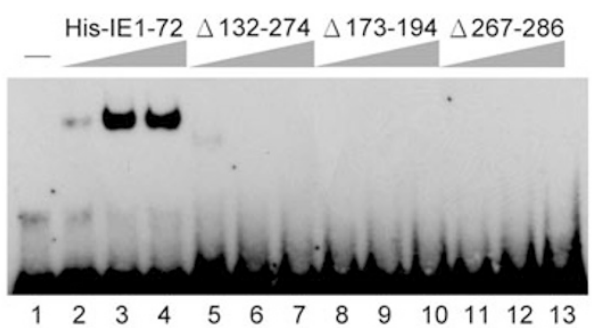

B
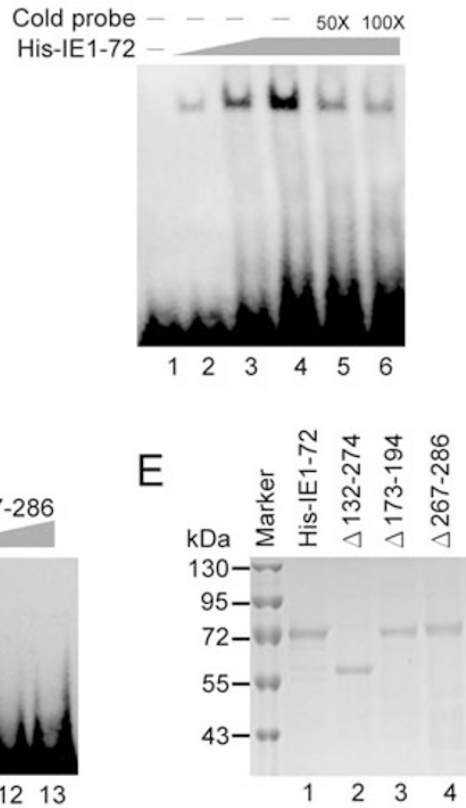

C

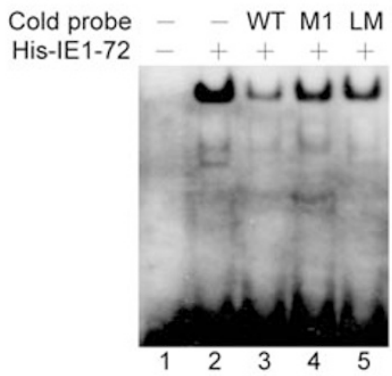

WT: TTCACCAATCGGCGGCCTCCACGACGG M1: TTCGTAAGCTTTAGGCCTCCACGACGG LM: TTCAGATCTCGGCGGCTCCACGACGG

Figure 5 IE1-72 binds to ERSE in vitro. E. coli-purified His-tagged IE1 (His-IE1-72) was mixed with the biotin end-labeled ERSE in the absence or presence of increasing amounts of His Ab (A, lane 5 to 7 ), wild-type ERSE competitor (B, lanes 5 and 6 ) or mutant ERSE competitor (C, lanes 4 and 5), followed by electrophoretic mobility shift assay (EMSA). (A) The amounts of IE1-72 added are as follows: lane 1, $0 \mu \mathrm{g}$; lane 2, $0.2 \mu \mathrm{g}$; lane 3, $0.5 \mu \mathrm{g}$; and lanes 4 to 7, $1 \mu \mathrm{g}$. (B) The amounts of IE1-72 added are as follows: lane 1, $0 \mu \mathrm{g}$; lane 2, $0.25 \mu \mathrm{g}$; lane 3, $1 \mu \mathrm{g}$; and lanes 4 to 6, $2 \mu \mathrm{g}$. Lanes 5 and 6 contain 50- and 100fold molar excess, respectively, of the wild-type ERSE competitor. (C) The amounts of IE1-72 added are as follows: lane 1, $0 \mu \mathrm{g}$; and lanes 2 to $5,1 \mu \mathrm{g}$. Lanes 3 to 5 contain a 200 -fold molar excess of biotin end-labeled wild-type ERSE (lane 3 ) or mutant ERSE as indicated. The nucleotide sequences of the probes used are shown below the gel. Mutated nucleotides are bolded and underlined. (D) IE1-72 mutants defective in grp78 promoter transactivation fail to bind to the ERSE. Increasing amounts of His-IE1-72 or the indicated mutants were added to each reaction. The amounts of IE1-72 and its mutants added are as follows: $0 \mu \mathrm{g}$ for lane $1 ; 0.25 \mu \mathrm{g}$ for lanes $2,5,8$ and $11 ; 1.5 \mu \mathrm{g}$ for lanes $3,6,9$ and 12 ; and $2.5 \mu \mathrm{g}$ for lanes $4,7,10$ and 13. (E) The 10\% SDS-PAGE shows equal expression of His-IE1-72 derivatives used in D. 

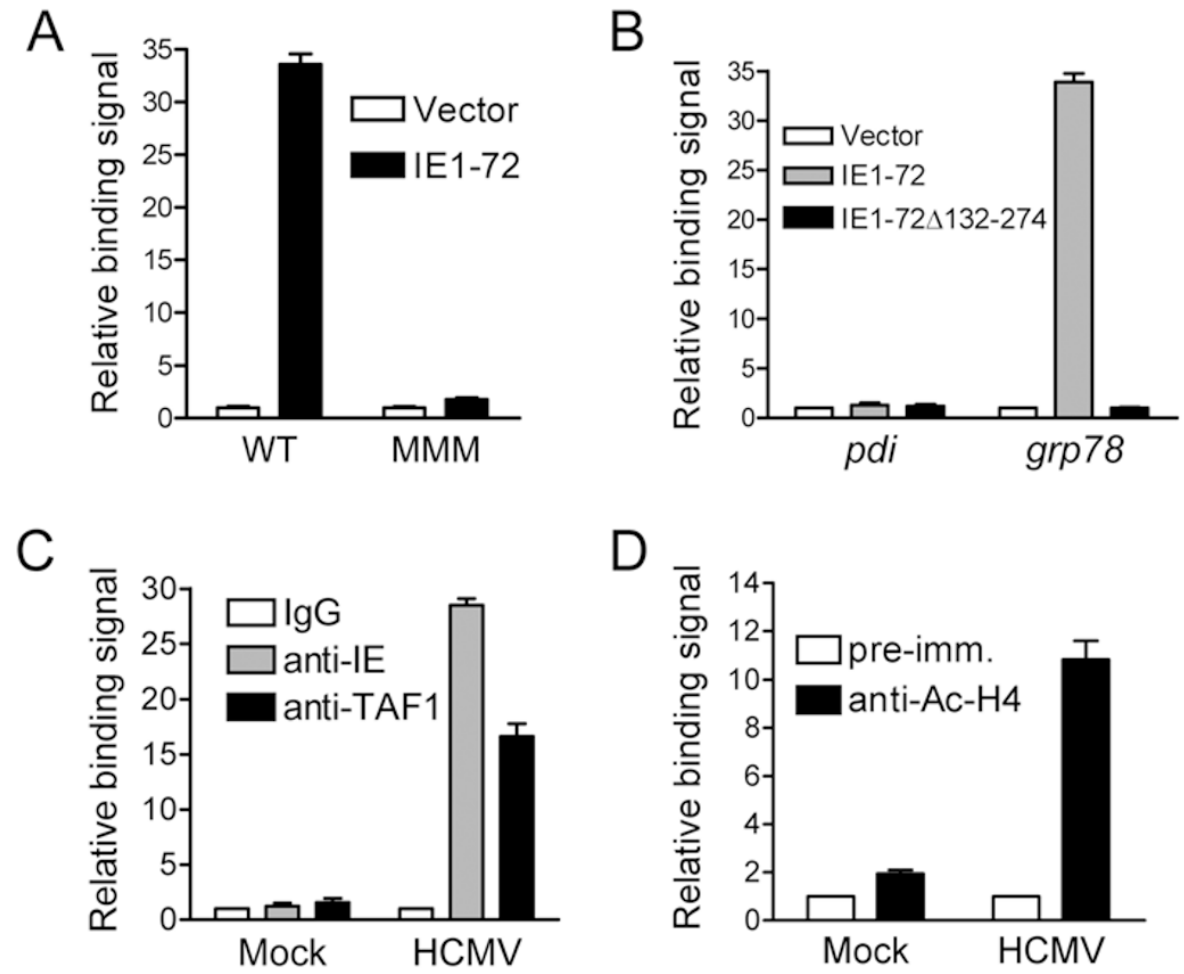

Figure 6 IE1-72 binding to the grp78 promoter accompanies TAF1 recruitment and local histone acetylation. (A) IE1-72 associates with the wild-type grp78 promoter but not the promoter with the mutated ERSE. U2OS cells were transfected with the plasmid encoding the firefly luciferase gene driven by the wild-type (WT) or mutated ERSE (MMM) promoter together with vector control (white bar) or plasmid encoding IE1-72 (black bar). After $24 \mathrm{~h}$, cells were harvested and subjected to ChIP analysis with an anti-IE antibody (mab810, Chemicon). The co-precipitated DNA was analyzed by quantitative PCR with primers amplifying only the exogenously added grp78 promoter. (B) IE1-72 associates with the grp78 promoter In vivo. Similar ChIP experiments were applied as in A, except that the binding of wild-type IE1-72 or its deletion mutant lacking aas 132 to 274 to the endogenous promoter of pdi or grp78 was analyzed. (C, D) HCMV infection co-induces the binding of viral IE proteins and TAF1 to the grp78 promoter (C) as well as local H4 hyper-acetylation. MRC-5 cells that were mock infected, or infected with HCMV at an MOI of 1, were subjected to ChIP assays at 2 d.p.i. with the indicated antibodies. The precipitated DNA was analyzed by quantitative PCR with primers amplifying the grp78 promoter region. Data in A, B, C and D are presented as the mean \pm SD from three independent assays.

shown in Figure 6C, both HCMV IE and TAF1 associated with the $\operatorname{grp} 78$ promoter at the ERSE-containing region. Notably, histone $\mathrm{H} 4$ associated with the same region of DNA was hyper-acetylated (Figure 6D), indicating that the grp 78 promoter in HCMV-infected cells adopted an open chromatin structure, and was transcriptionally active. Because the anti-IE Ab used in this study recognizes the conserved N-terminus of IE1-72 and IE286 , we could not distinguish whether the observed effect was from IE1-72 or IE2-86. To accomplish this, U2OS cells were transiently transfected with the plasmid encoding IE1-72 or IE2-86 and subjected to ChIP assay using the same anti-IE Ab. Supplementary information Figure $\mathrm{S} 1$ shows that the Ab pulled down the grp 78 promoter in cells expressing IE1-72 but not IE2-86. This result indicates that, although the HCMV IE Ab we used recognizes both IE1-72 and IE2-86, the grp78 promoter precipitated was only bound by IE1-72. The data also suggest that IE2-86 unlikely associates with the $\operatorname{grp} 78$ promoter in infected cells.

\section{Discussion}

This study identifies an essential role for IE1-72 in HCMV-mediated grp78 upregulation (Figure 2) via its direct binding to the $g r p 78$ promoter through the ERSE elements (Figures 3, 4 and 5). IE1-72 binding correlates with transcriptional initiation because TAF1, the largest component of $\mathrm{TF}_{\mathrm{II}} \mathrm{D}$, was concomitantly recruited to the promoter during viral infection (Figure 6). IE1-72 is known to regulate transcription by interacting with a variety of transcriptional factors or cofactors $[20,23,33-$ 
38], but whether it directly associates with DNA was unknown. Interestingly, we demonstrate in this report that IE1-72 indeed possesses DNA-binding ability (Figure 5). We show that the recombinantly purified IE1-72 binds to ERSE on the grp 78 promoter (Figure 5) and that the potential DNA-binding domain of IE1-72 was mapped to exon 4 from aa 132 to aa 286 (Figure 5). Mutant IE1-72 proteins lacking fragments within this region consistently failed to stimulate grp 78 promoter activity (Figure 3). Thus, our study provides a novel mechanism of IE1-72 regulation of gene expression.

In contrast to IE1-72, the splicing isoform IE2-86 did not seem to regulate grp 78 expression (Figure 2A and $2 \mathrm{~B}$ ), which is likely due to its inability to associate with the grp 78 promoter (Supplementary information, Figure S1). Although IE2-86 is known to bind to the cisrepression sequence (CRS) of the HCMV MIEP [39, 40], the CRS characterized as 5'-GC(N10)CG-3' with AT-rich sequences in $\mathrm{N}_{10}$ differs from ERSE.

We show in Figure 2D that knocking down IE1-72 almost abolished the HCMV-mediated GRP78 induction at 1 d.p.i.. This finding demonstrates an essential role of IE1-72 in this event at that time point. Nevertheless, whether IE1-72 is the only viral factor responsible for $\operatorname{grp} 78$ upregulation is not clear. The question can be partly addressed by comparing the time-course expression of GRP78 with IE1-72. Previous reports have shown that GRP78 protein level is increased at 24 h.p.i. [17, 41]. However, the authors did not provide the kinetic expression pattern of the grp 78 mRNA. Our investigation indicates that both grp 78 mRNA and IE1-72 protein were induced after 6 to 8 h.p.i. (Supplementary information, Figure S2), which supports our current finding that IE1-72 likely activates the grp 78 promoter. The delayed appearance of the GRP78 protein might be mediated by other viral or cellular factors at the post-transcriptional level. Indeed, there are reports describing factors that regulate GRP78 translation through the internal ribosome entry site [42-44], and the same explanation might be applied to the conflict in which the increase in $9 r p 78$ mRNA by IE1-72 transfection did not translate proportionally to an increase in the protein level (compare Figure $2 \mathrm{~A}$ with $2 \mathrm{~B}$ ). In addition, the two reports mentioned above show that the GRP78 protein level peaks from 2 to 3 d.p.i. and decreases thereafter [17, 41]. Given that the IE1-72 protein is known to be sustained at a high level throughout the viral life cycle [19], we strongly suspect that other viral or cellular factors contribute to orchestrating grp 78 expression during the late stage of HCMV infection. Alternatively, IE1-72 might be post-translationally modified at different stages, and this might alter its ability to regulate $\operatorname{grp} 78$ promoter function. These possibilities await further investigation.

In this study, we observed that HCMV infection stimulated the expression of grp 78, but not pdi (Figure $1 \mathrm{~A}$ and $1 \mathrm{~B}$ ), although both of them are ER chaperones, known to be induced by UPR [1]. Selective induction of GRP78 by HCMV implies unique roles of GRP78 in HCMV viral growth. Indeed, GRP78 has been reported to participate in HCMV virion assembly [16, 41]. Depletion of GRP78 interferes with the protein folding process in the ER, and this is thought to result in the accumulation of misfolded viral proteins, and hence, retards viral growth. Alternatively, the redistribution of the ER-Golgi apparatus during HCMV infection [45] might change the binding preference of GRP78 toward HCMV viral proteins. Buchkovich et al. [16] demonstrated that GRP78 binds to pp28, a late HCMV protein in the tegument layer, and TRS1, another HCMV protein that has multiple functions in transactivation, viral DNA replication and virion assembly and by binding to these proteins GRP78 contributes to the formation and maintenance of the viral assembly compartments. Our finding of IE1-72-mediated GRP78 regulation further indicates that HCMV actively controls cellular gene expression to benefit viral growth.

GRP78 also plays a role in tumorigenesis. Small interfering RNA (siRNA)-mediated knockdown of GRP78 sensitizes malignant glioma cells to temozolomide and celecoxib. Conversely, ectopic expression of GRP78 renders cells more resistant to drugs [46, 47]. Both phenomena suggest that GRP78 protects cancer cells from druginduced stress and cell death. HCMV infection has been detected in human malignant glioma cells in vivo [48], and IE1-72 overexpression in glioma tumor cells potentiates the oncogenic phenotype with the reduction of $\mathrm{Rb}$ and p53 and the activation of the PI3 kinase/AKT pathway [25]. Given that the luciferase reporter driven by the grp 78 promoter is activated in IE1-72-expressing glioblastoma U373MG cells (data not shown), whether the IE1-72-enhanced tumorigenic potential of these glioma cells is also mediated by GRP78 remains an interesting question.

All together, these results demonstrate the mechanism by which HCMV induces grp 78 expression. The novel finding that IE1-72 binds directly to the ERSE of the grp 78 promoter reveals a new mechanism of gene regulation by IE1-72.

\section{Materials and Methods}

\section{Cells and viruses}

Human lung fibroblast lines MRC-5 (ATCC, CCL-171) and HEL299 (ATCC, CCL-137), and osteosarcoma U2OS cells (ATCC, HTB-96) were cultured in $\alpha$ Minimum Essential Media ( $\alpha$-MEM, Invitrogen) and McCoy's 5A medium (Sigma), respectively, con- 
taining 10\% fetal bovine serum (FBS, Upstate), $100 \mathrm{U} / \mathrm{ml}$ penicillin and $100 \mu \mathrm{g} / \mathrm{ml}$ streptomycin (Gibco). HCMV RC256, a Towne strain derivative [49], was obtained from ATCC and propagated in MRC-5 cells for 4 to 12 passages, and the viral titer was determined by plaque assay. For infection, cells were incubated with growth medium containing $2 \%$ of FBS and virus at $37{ }^{\circ} \mathrm{C}$ for $2 \mathrm{~h}$ at a multiplicity of infection (MOI) indicated in each figure, and then replaced with normal growth medium.

\section{Plasmids}

The pSEP7-IE1-72 and pSEP7-IE2-86 mammalian expression vectors of IE1-72 and IE2-86, respectively, were described previously [50]. pJHA303 was used to express full-length IE1-72 (aa 1-491), pJHA308 for IE1-72 $\Delta 132-274$, pJHA346 for IE1-72 $\Delta 290$ 320 and pJHA423 for IE1-72 $\Delta 421-491$; all were provided by Dr JH Ahn [51]. The pSG5 plasmids encoding IE1-72 $\Delta 267-286$ and IE1-72 $\Delta 271-193$ were provided by Dr J Sinclair [20] and Dr JC Azizkhan [23], respectively. The pcDNA3-IE1-72-M12 plasmid encoding IE1-72 with base substitutions in exon 2 and the pcDNA3-IE1-72-M13-1 and M13-2 plasmids encoding IE1-72 with base substitutions in exon 3 were provided by Dr ES Huang [22]. The luciferase reporter plasmid containing the WT human grp 78 promoter from -134 to $+4 \mathrm{bp}$, or the mutant promoter with ERSEs substitutively mutated in CCAAT boxes (Figure 4A) was provided by Dr K Mori [10]. pRL-SV40 encoding the Renilla luciferase gene was from Promega. For purification of His-tagged IE1-72 derivatives, the corresponding cDNAs were PCR-amplified and cloned into pET100/D (Invitrogen). The oligonucleotides of the ERSE and its mutants illustrated in Figure 4B were synthesized by Purigo Biotech, annealed and cloned into the pGL3 promoter (Promega) between the Nhe I and Bgl II sites upstream of the SV40 promoter.

\section{Transfection, western blot, luciferase assay and RT-PCR}

All transfections were carried out with Lipofectamine 2000 (Invitrogen) according to the manufacturer's instructions. Western blot and luciferase assays were performed as described previously [52]. For RT-PCR, RNAs were harvested using TRIzol reagent (Invitrogen) and reversely transcribed to cDNA by SuperScript II RT (Invitrogen) according to the manufacturer's instructions. The cDNAs were analyzed on 2\% of agarose gels after 20-30 cycles of PCR using the following oligonucleotides: grp78, 5'-GCCGAGGAGGAGGACAAGAA-3' (forward) and 5'-CACAGACGGGTCATTCCACG-3' (reverse); $p d i, 5^{\prime}$-GGAGAACCTGCTGGACTTTATC-3' (forward) and 5'-GGCATAGAACTCCACAAAGACG-3' (reverse); $\beta$-actin, 5'-GCATCCACGAAACTACCT-3' (forward) and 5'-GAAAGGGTGTAACGCAAC-3' (reverse). The target cDNAs were quantitated by real-time PCR (LightCycler 480, Roche) according to the manufacturer's instruction.

\section{Antibodies}

The primary antibodies used in western blots are as follows: mouse anti-cytomegalovirus $\mathrm{Ab}$ against IE proteins of cytomegalovirus (mab810, Millipore), mouse anti- $\beta$-tubulin (mab5562, Millipore), mouse anti- $\beta$-actin (mab1501, Millipore), mouse anti-BiP/ GRP78 (BD biosciences), rabbit anti-PDI (GTX101468, GeneTex) and rabbit anti-HSP90B1 (GTX103232, GeneTex). Anti-His $\mathrm{Ab}$ (27-4710-01) for EMSA was obtained from GE Healthcare. The primary antibodies used in ChIP are as follows: mouse anti-
TAFII250 (6B3), mouse anti-cytomegalovirus Ab against IE proteins of cytomegalovirus (mab810) and rabbit anti-acetylated histone 4 (06866) (all from Millipore).

\section{Protein purification and electrophoretic mobility shift assay (EMSA)}

His-IE1-72 and its deletion mutants were expressed in and purified from the $E$. coli BL21(DE3)pLys strain (Novagen) with pET100/D-IE1-72 or pET100/D-IE1-72 $132-274, \Delta 173-194$ and $\Delta 267-286$, followed by purification with Ni Sepharose 6 Fast Flow beads (GE Healthcare) according to the manufacturer's instruction. Purified proteins were dialyzed against buffer C (10 mM Hepes, pH 7.2, $10 \%$ glycerol, $5 \mathrm{mM} \mathrm{MgCl}, 1 \mathrm{mM} \mathrm{MnCl}_{2}, 50 \mathrm{mM} \mathrm{KCl}$ ) and stored at $-80{ }^{\circ} \mathrm{C}$. His-IE1-72 was incubated with biotin endlabeled ERSE element (nucleotides from -65 to -39 ; Purigo Biotech) in a $20 \mu \mathrm{l}$ reaction of buffer $\mathrm{C}$ containing $1 \mu \mathrm{g}$ BSA and $4 \mu \mathrm{g}$ poly(deoxyinosinic-deoxycytidylic) acid sodium salt (poly(dI:dC), Sigma) at room temperature for $30 \mathrm{~min}$. For super-shift assays, increasing amounts of anti-His Ab were incubated with the protein/DNA mix. For competition assays, non-labeled probes were used as cold probes and mixed together with the biotin end-labeled probe prior to the addition of recombinant His-IE1-72. After incubation, samples were resolved by $6 \%$ of native polyacrylamide gels in $0.5 \times$ TBE buffer with $100 \mathrm{~V}$ for $2 \mathrm{~h}$. DNA resolved on the gel were transferred onto blotting membrane (Zeta-Probe GT Genomic Tested Blotting Membrane, Bio-Rad Laboratory) in 0.5× TBE buffer with $380 \mathrm{~mA}$ at $4{ }^{\circ} \mathrm{C}$ for 1 to $2 \mathrm{~h}$. The membrane was cross-linked by UV (Ultraviolet crosslinker, UVP LLC) and incubated with the blocking buffer $(25 \mathrm{mM} \mathrm{NaPi}, \mathrm{pH} 6.5,6 \times \mathrm{SSC}, 5 \times$ Dendard's solution, $200 \mathrm{ng} / \mathrm{ml}$ salmon sperm DNA and $0.5 \%$ SDS) at $37{ }^{\circ} \mathrm{C}$ for $30 \mathrm{~min}$. Horseradish peroxidase-conjugated streptavidin (Jackson ImmunoResearch Laboratories), diluted in $2 \times \mathrm{SSC}$ buffer $(300 \mathrm{mM}$ sodium chloride and $20 \mathrm{mM}$ sodium citrate, $\mathrm{pH}$ 7.0) containing $0.5 \% \mathrm{SDS}$, was mixed with the blot at room temperature for $30 \mathrm{~min}$ to detect the biotin-labeled DNA. The blot was extensively washed with $2 \times$ SSC containing $0.5 \%$ SDS and subsequently developed by enhanced chemiluminescence (GE Healthcare) according to the manufacturer's instruction.

\section{Chromatin immunoprecipitation assay}

ChIP assays were performed as described [50] with modifications. The final DNA samples were analyzed by real-time PCR (LightCycler 480, Roche) according to the manufacturer's instructions. The oligonucleotides amplifying the region containing three ERSEs and the TATA-box of the grp 78 promoter are 5'-CGGAGCAGTGACGTTTAT-3' (forward) and 5'-GTCGCCTACTCGGCTTAT-3' (reverse). Oligonucleotides for the pdi promoter are 5'-GCAGAGTCCGTGCTACCGAAA-3' (forward) and 5'-TCTCATTGGCTCCCGACAAGA-3' (reverse). Oligonucleotides for both WT and the ERSE-mutated grp 78 promoter on pGL3-basic reporters are 5'-ATAAGCCGAGTAGGCGAC-3' (forward) and 5'GTTCCATCTTCCAGCGGATAG-3' (reverse).

\section{Knockdown of IE1-72 expression}

siRNA against IE1-72 was designed and synthesized by Ambion. For si-IE1-72: 5'-GGAUGUUUGCAGAAUGCCUtt-3' (sense) and 5'-AGGCAUUCUGCAAACAUCCtc-3' (antisense). For siScramble: 5'-AGTACTGCTTACGATACGGtt-3' (sense) and 5'-CCGTATCGTAAGCAGTACTtc-3' (antisense). In brief, cells at 
$80 \%$ confluence in a $10-\mathrm{cm}$ dish were transiently transfected with $20 \mathrm{nM}$ of siRNA by Lipofectamine 2000, and the medium was replaced after overnight incubation. Cells were transfected with the same siRNA again at $24 \mathrm{~h}$ after the first transfection. At $48 \mathrm{~h}$ after the second transfection, cells were subcultured into 6-well plates at a density of $5 \times 10^{5}$ cells per well; these cells were then mock infected or infected with HCMV at an MOI of 1 to 2 the following day and subjected to western blot analysis at 24 h.p.i.

\section{Acknowledgments}

We thank Drs ES Huang, K Mori, JH Ahn and JH Sinclair for providing plasmids and SC Chen for constructing plasmids pSEP7-IE1-72 and pSEP-7IE2-86. The study was supported by grants from Acadmia Sinica to LJJ.

\section{References}

1 Lee AS. The glucose-regulated proteins: stress induction and clinical applications. Trends Biochem Sci 2001; 26:504-510.

2 Fink AL. Chaperone-mediated protein folding. Physiol Rev 1999; 79:425-449.

3 Bertolotti A, Zhang Y, Hendershot LM, Harding HP, Ron D. Dynamic interaction of BiP and ER stress transducers in the unfolded-protein response. Nat Cell Biol 2000; 2:326-332.

4 Okada T, Yoshida H, Akazawa R, Negishi M, Mori K. Distinct roles of activating transcription factor 6 (ATF6) and doublestranded RNA-activated protein kinase-like endoplasmic reticulum kinase (PERK) in transcription during the mammalian unfolded protein response. Biochem J 2002; 366:585-594.

5 Shen J, Chen X, Hendershot L, Prywes R. ER stress regulation of ATF6 localization by dissociation of BiP/GRP78 binding and unmasking of Golgi localization signals. Dev Cell 2002; 3:99-111.

6 Harding HP, Zhang Y, Ron D. Protein translation and folding are coupled by an endoplasmic-reticulum-resident kinase. $\mathrm{Na}$ ture 1999; 397:271-274.

7 Yoshida H, Matsui T, Yamamoto A, Okada T, Mori K. XBP1 mRNA is induced by ATF6 and spliced by IRE1 in response to ER stress to produce a highly active transcription factor. Cell 2001; 107:881-891.

8 Mori K. Signaling pathways in the unfolded protein response: development from yeast to mammals. J Biochem 2009; 146:743-750.

9 Li M, Baumeister P, Roy B, et al. ATF6 as a transcription activator of the endoplasmic reticulum stress element: thapsigargin stress-induced changes and synergistic snteractions with NF-Y and YY1. Mol Cell Biol 2000; 20:5096-5106.

10 Yoshida H, Haze K, Yanagi H, Yura T, Mori K. Identification of the cis-acting endoplasmic reticulum stress response element responsible for transcriptional induction of mammalian glucose-regulated proteins. Involvement of basic leucine zipper transcription factors. J Biol Chem 1998; 273:3374133749.

11 Roy B, Li WW, Lee AS. Calcium-sensitive transcriptional activation of the proximal CCAAT regulatory element of the grp78/BiP promoter by the human nuclear factor CBF/NF-Y. J Biol Chem 1996; 271:28995-29002.

12 Abdelrahim M, Liu S, Safe S. Induction of endoplasmic retic- ulum-induced stress genes in Panc-1 pancreatic cancer cells is dependent on Sp proteins. J Biol Chem 2005; 280:1650816513.

13 Parker R, Phan T, Baumeister P, et al. Identification of TFII-I as the endoplasmic reticulum stress response element binding factor ERSF: its autoregulation by stress and interaction with ATF6. Mol Cell Biol 2001; 21:3220-3233.

14 Mayer MP. Recruitment of Hsp70 chaperones: a crucial part of viral survival strategies. Rev Physiol Biochem Pharmacol 2005; 153:1-46.

15 Tardif KD, Waris G, Siddiqui A. Hepatitis C virus, ER stress, and oxidative stress. Trends Microbiol 2005; 13:159-163.

16 Buchkovich NJ, Maguire TG, Paton AW, Paton JC, Alwine JC. The endoplasmic reticulum chaperone BiP/GRP78 is important in the structure and function of the HCMV assembly compartment. J Virol 2009; 83:11421-11428.

17 Isler JA, Skalet AH, Alwine JC. Human cytomegalovirus infection activates and regulates the unfolded protein response. J Virol 2005; 79:6890-6899.

18 Landolfo S, Gariglio M, Gribaudo G, Lembo D. The human cytomegalovirus. Pharmacol Ther 2003; 98:269-297.

19 Mocaski ES, Shenk T, Pass RF. Cytomegalovirus: replication. In: Knipe DM, Howley PM, eds. Fields Virology. Philadelphia: Lippincott Williams and Wilkins, 2007:2718-2727.

20 Hayhurst GP, Bryant LA, Caswell RC, Walker SM, Sinclair JH. CCAAT box-dependent activation of the TATA-less human DNA polymerase alpha promoter by the human cytomegalovirus 72-kilodalton major immediate-early protein. $J$ Virol 1995; 69:182-188.

$21 \mathrm{Xu} \mathrm{Y,} \mathrm{Ahn} \mathrm{J-H,} \mathrm{Cheng} \mathrm{M,} \mathrm{et} \mathrm{al.} \mathrm{Proteasome-independent} \mathrm{dis-}$ ruption of PML oncogenic domains (PODs), but not covalent modification by SUMO-1, is required for human cytomegalovirus immediate-early erotein IE1 to inhibit PML-mediated transcriptional repression. J Virol 2001; 75:10683-10695.

22 Zhang Z, Huong SM, Wang X, Huang DY, Huang ES. Interactions between human cytomegalovirus IE1-72 and cellular p107: functional domains and mechanisms of up-regulation of cyclin E/cdk2 kinase activity. J Virol 2003; 77:12660-12670.

23 Pajovic S, Wong EL, Black AR, Azizkhan JC. Identification of a viral kinase that phosphorylates specific E2Fs and pocket proteins. Mol Cell Biol 1997; 17:6459-6464.

24 Kim S, Yu SS, Lee IS, et al. Human cytomegalovirus IE1 protein activates AP-1 through a cellular protein kinase(s). J Gen Virol 1999; 80:961-969.

25 Cobbs CS, Soroceanu L, Denham S, Zhang W, Kraus MH. Modulation of oncogenic phenotype in human glioma cells by cytomegalovirus IE1-mediated mitogenicity. Cancer Res 2008; 68:724-730.

26 Cobbs CS, Soroceanu L, Denham S, et al. Human cytomegalovirus induces cellular tyrosine kinase signaling and promotes glioma cell invasiveness. $J$ Neurooncol 2007; 85:271280.

27 Cobbs CS, Harkins L, Samanta M, et al. Human cytomegalovirus infection and expression in human malignant glioma. Cancer Res 2002; 62:3347-3350.

28 Nevels M, Brune W, Shenk T. SUMOylation of the human cytomegalovirus 72-kilodalton IE1 protein facilitates expression of the 86-kilodalton IE2 protein and promotes viral replication. $J$ Virol 2004; 78:7803-7812. 
29 Hwang S, Gou Z, Kuznetsov IB. DP-bind: a web server for sequence-based prediction of DNA-binding residues in DNAbinding proteins. Bioinformatics 2007; 23:634-636.

30 Kuznetsov IB, Gou Z, Li R, Hwang S. Using evolutionary and structural information to predict DNA-binding sites on DNA-binding proteins. Proteins 2006; 64:19-27.

31 Lukac DM, Harel NY, Tanese N, Alwine JC. TAF-like functions of human cytomegalovirus immediate-early proteins. $J$ Virol 1997; 71:7227-7239.

32 Mizzen CA, Yang X-J, Kokubo T, et al. The TAFII250 subunit of TFIID has histone acetyltransferase activity. Cell 1996; 87:1261-1270.

33 Johnson RA, Yurochko AD, Poma EE, Zhu L, Huang ES. Domain mapping of the human cytomegalovirus IE1-72 and cellular p107 protein-protein interaction and the possible functional consequences. J Gen Virol 1999; 80:1293-1303.

34 Margolis MJ, Pajovic S, Wong EL, et al. Interaction of the 72-kilodalton human cytomegalovirus IE1 gene product with E2F1 coincides with E2F-dependent activation of dihydrofolate reductase transcription. J Virol 1995; 69:7759-7767.

35 Poma EE, Kowalik TF, Zhu L, Sinclair JH, Huang ES. The human cytomegalovirus IE1-72 protein interacts with the cellular p107 protein and relieves p107-mediated transcriptional repression of an E2F-responsive promoter. J Virol 1996; 70:7867-7877.

36 Huh YH, Kim YE, Kim ET, et al. Binding STAT2 by the acidic domain of human cytomegalovirus IE1 promotes viral growth and is negatively regulated by SUMO. J Virol 2008; 82:10444-10454.

37 Nevels M, Paulus C, Shenk T. Human cytomegalovirus immediate-early 1 protein facilitates viral replication by antagonizing histone deacetylation. Proc Natl Acad Sci U S A 2004; 101:17234-17239.

38 Yurochko AD, Mayo MW, Poma EE, Baldwin AS, Jr, Huang ES. Induction of the transcription factor $\mathrm{Sp} 1$ during human cytomegalovirus infection mediates upregulation of the p65 and p105/p50 NF-kappaB promoters. J Virol 1997; 71:46384648 .

39 Cherrington JM, Khoury EL, Mocarski ES. Human cytomegalovirus ie 2 negatively regulates alpha gene expression via a short target sequence near the transcription start site. $J$ Virol 1991; 65:887-896.

40 Jupp R, Hoffmann S, Depto A, et al. Direct interaction of the human cytomegalovirus IE86 protein with the cis repression signal does not preclude TBP from binding to the TATA box. $J$ Virol 1993; 67:5595-5604.
41 Buchkovich NJ, Maguire TG, Yu Y, et al. Human cytomegalovirus specifically controls the levels of the endoplasmic reticulum chaperone $\mathrm{BiP} / \mathrm{GRP} 78$, which is required for virion assembly. J Virol 2008; 82:31-39.

42 Cho S, Park SM, Kim TD, et al. BiP internal ribosomal entry site activity is controlled by heat-induced interaction of NSAP1. Mol Cell Biol 2007; 27:368-383.

43 Kim YK, Back SH, Rho J, Lee SH, Jang SK. La autoantigen enhances translation of BiP mRNA. Nucleic Acids Res 2001; 29:5009-5016.

44 Yang Q, Sarnow P. Location of the internal ribosome entry site in the $5^{\prime}$ non-coding region of the immunoglobulin heavychain binding protein (BiP) mRNA: evidence for specific RNA-protein interactions. Nucleic Acids Res 1997; 25:28002807.

45 Das S, Vasanji A, Pellett PE. Three-dimensional structure of the human cytomegalovirus cytoplasmic virion assembly complex includes a reoriented secretory apparatus. $J$ Virol 2007; 81:11861-11869.

46 Kardosh A, Golden EB, Pyrko P, et al. Aggravated endoplasmic reticulum stress as a basis for enhanced glioblastoma cell killing by bortezomib in combination with celecoxib or its non-coxib analog, 2,5-dimethyl-celecoxib. Cancer Res 2008; 68:843-851.

47 Pyrko P, Schonthal AH, Hofman FM, Chen TC, Lee AS. The unfolded protein response regulator GRP78/BiP as a novel target for increasing chemosensitivity in malignant gliomas. Cancer Res 2007; 67:9809-9816.

48 Cobbs CS, Harkins L, Samanta M, et al. Human cytomegalovirus infection and expression in human malignant glioma. Cancer Res 2002; 62:3347-3350.

49 Spaete RR, Mocarski ES. Insertion and deletion mutagenesis of the human cytomegalovirus genome. Proc Natl Acad Sci USA 1987; 84:7213-7217.

50 Hsu CH, Chang MD, Tai KY, et al. HCMV IE2-mediated inhibition of HAT activity downregulates p53 function. $E M B O$ J 2004; 23:2269-2280.

51 Lee HR, Kim DJ, Lee JM, et al. Ability of the human cytomegalovirus IE1 protein to modulate sumoylation of PML correlates with its functional activities in transcriptional regulation and infectivity in cultured fibroblast cells. J Virol 2004; 78:6527-6542.

52 Juan LJ, Shia WJ, Chen MH, et al. Histone deacetylases specifically down-regulate $\mathrm{p} 53$-dependent gene activation. $J$ Biol Chem 2000; 275:20436-20443.

(Supplementary information is linked to the online version of the paper on the Cell Research website.) 